\title{
Vibration-induced nanoscale friction modulation on piezoelectric materials
}

\author{
Jiawei CAO ${ }^{1}$, Qunyang LI ${ }^{1,2, *}$ \\ ${ }^{1}$ Applied Mechanics Laboratory, Department of Engineering Mechanics, Tsinghua University, Beijing 100084, China \\ ${ }^{2}$ State Key Laboratory of Tribology, Tsinghua University, Beijing 100084, China \\ Received: 26 April 2021 / Revised: 23 June 2021 / Accepted: 01 September 2021 \\ (C) The author(s) 2021.
}

\begin{abstract}
Mechanical vibration, as an alternative of application of solid/liquid lubricants, has been an effective means to modulate friction at the macroscale. Recently, atomic force microscopy (AFM) experiments and model simulations also suggest a similar vibration-induced friction reduction effect for nanoscale contact interfaces, although an additional external vibration source is typically needed to excite the system. Here, by introducing a piezoelectric thin film along the contact interface, we demonstrate that friction measured by a conductive AFM probe can be significantly reduced (more than $70 \%$ ) when an alternating current (AC) voltage is applied. Such real-time friction modulation is achieved owing to the localized nanoscale vibration originating from the intrinsic inverse piezoelectric effect, and is applicable for various material combinations. Assisted by analysis with the Prandtl-Tomlinson (P-T) friction model, our experimental results suggest that there exists an approximately linear correlation between the vibrational amplitude and the relative factor for perturbation of sliding energy corrugation. This work offers a viable strategy for realizing active friction modulation for small-scale interfaces without the need of additional vibration source or global excitation that may adversely impact device functionalities.
\end{abstract}

Keywords: vibration; friction modulation; piezoelectric materials; contact resonance; Prandtl-Tomlinson (P-T) model

\section{Introduction}

Friction widely exists in nature and in engineering mechanisms [1]. The ability to control friction, especially in real-time, is a goal that people have pursued for a long time in history [2]. With miniaturization of machines and devices, the surface-to-volume ratio of the mechanical systems has increased dramatically, which leads to prominent surface problems relating to friction and wear [3]. Although liquid lubricants have been widely adopted to reduce friction for macroscale interfaces [4], they are less applicable for small scale applications due to potential surface contamination and excessive viscosity of liquid [5]. Recently, twodimensional layered materials have been proposed as potential atomically-thin solid lubricants; however, to maintain superior lubrication performance, strict requirements, such as crystalline orientation, surface cleanliness, or even strain state, are often needed [6-9]. Therefore, how to effectively control friction and wear for micro- and nano-scale contact interfaces remains a grand challenge for material scientists and mechanicians.

Besides lubricants, mechanical vibration has been another common approach for controlling macroscopic friction [10, 11]. Recent experimental [12-14], theoretical [15-17], and molecular dynamics simulation [18, 19] results have demonstrated that mechanical vibration can achieve similar friction regulation even for smallscale contact interfaces. Using atomic force microscopy (AFM), Socoliuc et al. [12] showed that, by imposing an alternating current (AC) voltage between a conductive probe and an ionic crystal, friction could be substantially

* Corresponding author: Qunyang LI, E-mail: qunyang@tsinghua.edu.cn 
reduced owing to vibration caused by alternating electrostatic force. Similar friction modulation has also been achieved by directly vibrating the base of the AFM probe $[20,21]$, or the sample using external transducers [22-26]. The effect of mechanical vibration on microscopic friction is commonly understood by the modified Prandtl-Tomlinson (P-T) model [27-29], where the mechanical vibration is considered as a perturbation to the interaction corrugation associated with sliding. Despite their limited success, these theoretical models do not provide a clear correlation between the vibration amplitude and the magnitude of perturbation, which is essential for quantitative friction regulation. Furthermore, although nanoscale friction reduction has been demonstrated experimentally, the vibration driving methods so far are not easily implementable for practical applications. For instance, the alternating electrostatic force relies on the complex electrostatic interaction between the AFM probe and the sample $[12,14,20]$, which is closely related to the dielectric constant and surface potential of the sample, and geometries of the system [30]; while the other schemes require vibration of the whole contact interface using external actuators [20-26], which may introduce adverse impacts on functioning of the device. Therefore, it would be highly desirable if one can tune friction by vibrating the contact interface locally in a simple way.

In this work, nanoscale friction on piezoelectric thin films was measured by conductive AFM probes with an AC voltage imposed between the probes and the thin film substrates. The experimental results demonstrated that the local out-of-plane vibration induced by the inverse piezoelectric effect under a relative low voltage $(<10 \mathrm{~V})$ could be strong enough to significantly reduce

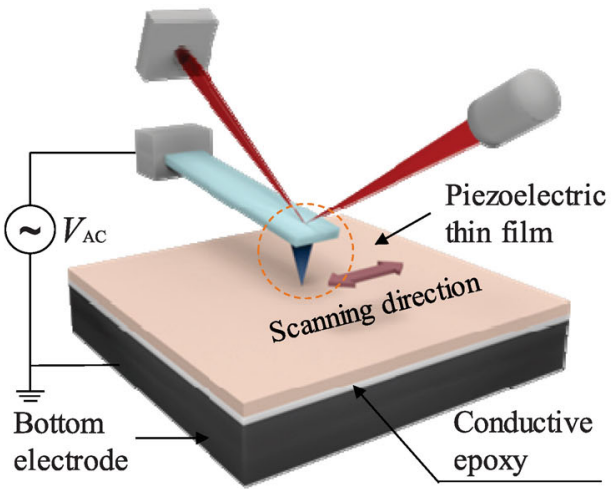

sliding friction if contact resonance of the system was exploited. Moreover, assisted by quantitative measurements and $\mathrm{P}-\mathrm{T}$ model simulations, an approximately linear dependence between the vibration amplitude and the magnitude of energy perturbation was established.

\section{Materials and experimental method}

Figure 1 depicts a schematic diagram of the experimental setup. In a commercial AFM system (NTegra, NT-MDT Inc., Russia), we measured friction of a conductive probe sliding on various piezoelectric thin films under the combined mode of lateral force microscopy (LFM) and piezoresponse force microscopy (PFM). Essentially, friction was measured via the conventional LFM while an AC bias voltage $\left(V_{\mathrm{AC}}\right)$ was additionally imposed between the probe and the bottom electrode beneath the piezoelectric film. Upon the introduction of $V_{\mathrm{AC}}$, the local region of the piezoelectric sample within the sliding contact area that is underneath the tip would deform periodically due to the inverse piezoelectric effect [31], resulting in vibration of the interface. Because of the decaying nature of the electric field, deformation occurs mostly within a relatively small region, typically a couple of microns directly below the tip apex [32]. In the friction experiments, by selecting relatively large normal loads, we ensured that the external force exerted by the cantilever would still be compressive even when the interface was vibrating. During the entire sliding process, we continuously monitored the lateral force signals, and collected the flexural amplitude and torsional amplitude of the probe using a lock-in amplifier. Friction force was determined by calculating

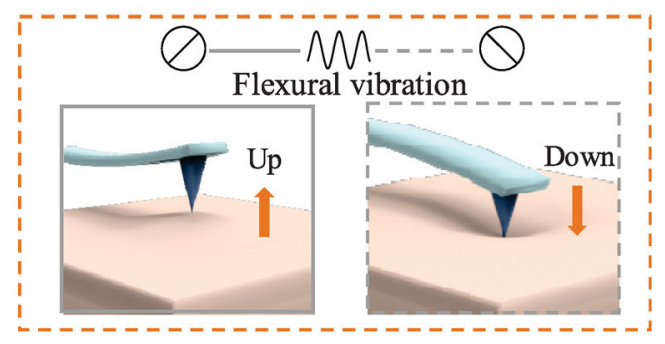

Fig. 1 A schematic diagram showing the experimental setup for friction measurement under vibration actuation stemming from the inverse piezoelectric effect of the piezoelectric thin film. 
the half of the difference of the trace and retrace lateral force signals. The lateral force was calibrated using a diamagnetic levitation spring system [33]. It is worth noting that the flexural signal in an AFM system reflects the angular deflection of the cantilever at the laser spot location. Therefore, for quasi-static AFM measurements, the flexural signal is linearly dependent on the out-of-plane displacement of the tip. However, for dynamic measurements, the relationship between the flexural signal and the out-of-plane displacement critically depends on the flexural vibration mode of the cantilever [34, 35], which hinders accurate experimental calibration of vibration amplitude signals, and will be further discussed in due course. In contrast, the correlation between the torsional angle of the cantilever and the lateral displacement of the tip primarily depends on the height of the tip, which is identical for quasi-static and dynamic measurements.

Unless otherwise specified, the friction experiments were performed on single-crystalline periodically poled lithium niobate (PPLN, Asylum Research Inc., USA) with a dimension of $3 \mathrm{~mm} \times 3 \mathrm{~mm} \times 500 \mu \mathrm{m}$, mounted on a metal substrate by conductive epoxy. Domains of the PPLN sample penetrate in the depth direction with spontaneous polarization perpendicular to the surface. To explore the applicability of the friction regulation strategy, we also conducted friction experiments on $(1-x) \mathrm{Pb}\left(\mathrm{Mg}_{1 / 3} \mathrm{Nb}_{2 / 3}\right) \mathrm{O}_{3}-x \mathrm{PbTiO}_{3}$ (PMN-PT, Shanghai Institute of Ceramics, China) sample with a dimension of $6 \mathrm{~mm} \times 6 \mathrm{~mm} \times 1 \mathrm{~mm}$, fixed on a metal substrate by conductive tape. Two types of commercial silicon probes, HA_C/ $\mathrm{W}_{2} \mathrm{C}+$ (wear-resistant $\mathrm{W}_{2} \mathrm{C}$ coating, ScanSens Inc., Germany) and ASYELEC-01-R2 (Ti/Ir coating, Asylum Research Inc., USA), were used. The typical spring constant and the first-order resonance frequency in the air $\left(f_{0}\right)$ were $0.65 \mathrm{~N} / \mathrm{m}, 37 \mathrm{kHz}$ for the HA_C $/ \mathrm{W}_{2} \mathrm{C}+$ probes, and $2.8 \mathrm{~N} / \mathrm{m}, 75 \mathrm{kHz}$ for the ASYELEC-01-R2 probes, respectively. All the experiments were conducted in ambient conditions $\left(20-25^{\circ} \mathrm{C}\right.$, relative humidity $30 \%-40 \%$ ).

\section{Results and discussion}

\subsection{Friction vs. actuation frequency}

We first conducted friction experiments on a single domain surface of PPLN $(100 \mathrm{~nm} \times 100 \mathrm{~nm})$ using an
HA_C $/ \mathrm{W}_{2} \mathrm{C}+$ probe under a normal load of $50 \mathrm{nN}$ and a scanning speed of $200 \mathrm{~nm} / \mathrm{s}$. The adhesion force was determined by the force-distance curve to be in the range of $7-7.6 \mathrm{nN}$. During the friction test, the AC voltage amplitude was fixed at $6 \mathrm{~V}$ while its frequency was slowly ramped from 1 to $800 \mathrm{kHz}$ with an incremental step of $1 \mathrm{kHz}$ per scan line. The contact resonance curve and thus conductivity of the probe did not change much after repeated scanning, suggesting negligible wear of the probe. As revealed by the topographic image shown in Fig. S1 in the Electronic Supplementary Material (ESM), the surface of the PPLN surface is rather smooth with a root-mean-square (RMS) roughness of $0.05 \mathrm{~nm}$. Variations of the flexural vibration amplitude, torsional vibration amplitude, and friction with the AC actuation frequency are shown in Fig. 2(a). As the actuation frequency sweeps from low to high, one can clearly observe three peaks in the vibration amplitude curves, which correspond to the first-order flexural contact resonance $\left(f_{\mathrm{n} 1}, 151 \mathrm{kHz}\right)$, the first-order torsional contact resonance $\left(f_{\mathrm{t}} 431 \mathrm{kHz}\right)$, and the second-order flexural contact resonance $\left(f_{\mathrm{n} 2}\right.$ $453 \mathrm{kHz}$ ). It should be noted that $f_{\mathrm{nl}}$ is 4.1 times higher than the first-order free flexural resonance $\left(f_{0}\right)$ due to extra contact interaction between the tip and the sample surface [36]. Accordingly, friction is found to be reduced by $83 \%, 76 \%$, and $92 \%$ at these three contact resonances compared to the mean value of friction at off-resonance frequencies. As no friction reduction was observed at half of the flexural resonances, vibration was unlikely to be caused by the capacitive force $[12,14,20]$. The PPLN sample is insulating and chemically stable and the metal elements (e.g., W and Ir) in the conductive coatings of probes do not react with air and water at room temperature, so electrochemical reaction was unlikely to occur in our friction measurements. Therefore, our experimental results directly confirm that local vibration originating from the intrinsic piezoelectricity of the sample film can be significant enough to effectively modulate friction at the nanoscale. We have also conducted repetitive experiments on opposite-oriented domains and obtained similar results. It should be mentioned that although the vibration induced by input electric energy can reduce frictional dissipation, there is no direct energy conversion between them.

Based on the polarization orientation of the PPLN 
(a)

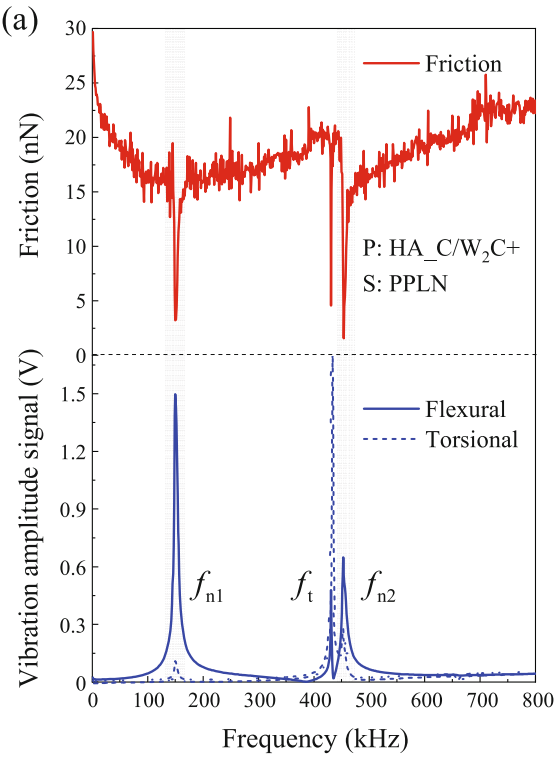

(b)

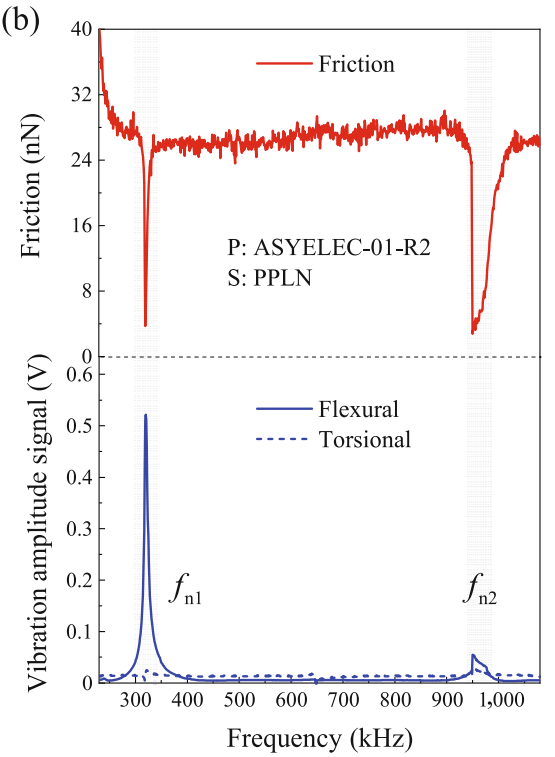

(c)

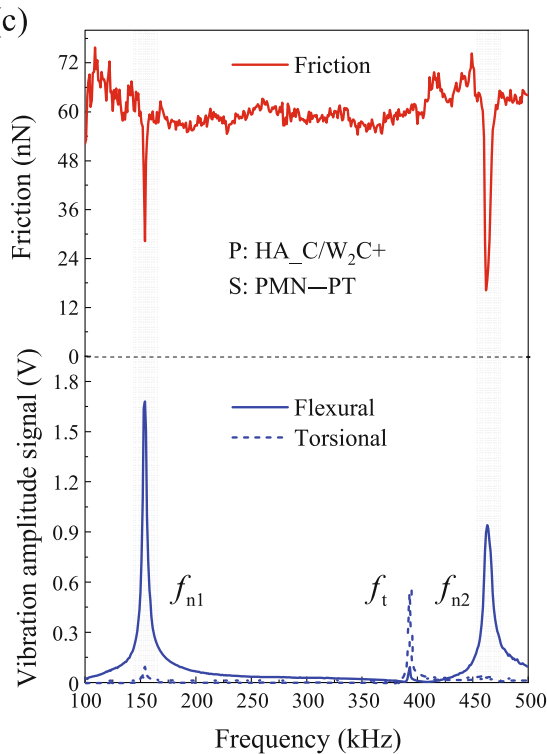

Fig. 2 Variations of friction and vibration amplitude signals with actuation frequency. (a) $\mathrm{HA} C / \mathrm{W}_{2} \mathrm{C}+$ probe on PPLN sample, with scan size of $100 \mathrm{~nm} \times 100 \mathrm{~nm}$, AC bias of $6 \mathrm{~V}$, and normal load of $50 \mathrm{nN}$; (b) ASYELEC-01-R2 probe on PPLN sample, with scan size of $100 \mathrm{~nm} \times 100 \mathrm{~nm}, \mathrm{AC}$ bias of $6 \mathrm{~V}$, and normal load of $208 \mathrm{nN}$; and (c) HA_C $/ \mathrm{W}_{2} \mathrm{C}+$ probe on PMN-PT sample, with scan size of $50 \mathrm{~nm} \times 50 \mathrm{~nm}$, AC bias of $5 \mathrm{~V}$, and normal load of $115 \mathrm{nN}$.

sample, actuation of the system was expected to occur primarily along the vertical direction, which would lead to flexural deformation of the cantilever. However, the crosstalk effect between the flexural and torsional deformation modes due to asymmetric geometry of the probe and/or asymmetric probe installation [20] can easily excite the torsional vibration mode in the experiment. However, according to previous experimental results with electrostatic actuation $[12,14,20]$ and theoretical study [37], friction typically would not be affected by lateral perturbation except in rare cases. Therefore, we speculated that the significant friction reduction at $f_{\mathrm{t}}$ in Fig. 2(a) was caused by the torsion-induced flexural vibration rather than the torsional deformation itself. To verify this hypothesis, we conducted similar friction experiment with a new probe, whose torsional resonance frequency $f_{\mathrm{t}}$ was shifted to $415 \mathrm{kHz}$ exhibiting a much weaker coupling between flexural and torsional modes. As shown in Fig. S2 in the ESM, when the crosstalk was suppressed, no substantial friction reduction was observed at $f_{\mathrm{t}}$.

Since the flexural vibration is essential for friction reduction, we proceeded to estimate its amplitude. For an AFM probe under local excitation of the piezoelectric vibration, the amplitude of its vertical displacement $A_{\mathrm{n}}$ at resonance can be estimated by

$$
A_{\mathrm{n}}=d_{33}^{\text {eff }} V_{\mathrm{AC}} Q
$$

where $d_{33}^{\text {eff }}$ is the "effective" inverse piezoelectric coefficient of the sample, $V_{\mathrm{AC}}$ is the AC bias amplitude, and $Q$ is the quality factor at the contact resonance. For typical PPLN material, $d_{33}^{\text {eff }}$ is $7.5-27 \mathrm{pm} / \mathrm{V}[38,39]$ and the quality factor $Q$ is estimated to be around 36.4 by fitting the peak of $f_{\mathrm{nl}}$ in Fig. 2(a) using a simple harmonic oscillator (SHO) model [40]. The resultant vertical vibration amplitude at resonance is expected to be around 1.6-5.9 $\mathrm{nm}$. Therefore, our experiment demonstrates that locally vibrating the interface within the nanoscale contact region even for a few nanometers could effectively suppress the sliding friction. However, it should be noted that, when the contact interface was excited at off-resonance, the vertical amplitude (estimated to be $45-162 \mathrm{pm}$ ) was not large enough to affect friction.

To test the universality of the above strategy for friction modulation, we carried out friction experiments with different probe materials and piezoelectric samples. Two more sets of data are shown in Figs. 2(b) and 2(c). We consistently found that as long as the vibration amplitude stemming from the piezoelectricity was properly amplified by contact resonance up to a few nanometers, surface friction 
could be reduced by $>70 \%$ at a relatively low voltage amplitude $(<10 \mathrm{~V})$.

\subsection{Friction vs. actuation amplitude}

Previous experiments and simulations [12, 22, 27, 28] have suggested that vibration-induced friction reduction is dependent on the magnitude of vibration amplitude. This is also consistent with the results shown in Fig. 2, where friction is significantly reduced only when the excitation amplitude is amplified via resonance. In order to further quantify the correlation between friction and vibration amplitude signal, we gradually ramped up the AC amplitude and monitored the variations of friction and flexural amplitude signal when the frequency was set at the two flexural resonances, i.e., $f_{\mathrm{nl}}$ and $f_{\mathrm{n} 2}$. As shown in Fig. 3(a), the flexural amplitude signal increases linearly with the AC amplitude for both frequencies but their slopes are noticeably different. For example, when the $\mathrm{AC}$ ramps from 0 to $9 \mathrm{~V}$, the flexural amplitude signal at $f_{\mathrm{n} 1}$ and $f_{\mathrm{n} 2}$ increases from nearly 0 to 1.6 and $0.6 \mathrm{~V}$, respectively. Despite the different change rates in flexural amplitude signal, the drop rate in friction with increasing $\mathrm{AC}$ amplitude is similar at both frequencies (from around 38 to 8 and $13 \mathrm{nN}$, respectively). When friction is plotted as a function of flexural amplitude signal in Fig. 3(b), one can see that friction decreases linearly with flexural amplitude signal under both actuation frequencies but their slopes differ by a factor of about 2.1. Such an abnormal dependency of friction reduction rate on vibration frequency has never been reported previously.
As explained in the introduction, the flexural signal recorded by the AFM system is a measure of change in inclination slope of the AFM micro-cantilever [35]. The correlation factor between the flexural signal and the vertical tip displacement, known as optical lever sensitivity (OLS), may depend on the vibration mode for dynamic measurements. It is possible that the real flexural amplitudes at first two resonant frequencies are actually similar and the different friction reduction rates might originate from the change in OLS for different vibration modes. To validate this hypothesis, we carried out finite element method (FEM) simulation to calculate the vibration modes of the micro-cantilever. The FEM model was constructed using the commercial FEM software (ABAQUS, Dassault Systemes Inc., France) based on the geometry of the AFM cantilever used in the experiment as shown in Fig. 4(a) (more details can be found in ESM).

Figure 4(b) shows the first-order and the secondorder flexural resonant modes of the cantilever. Because the OLS is proportional to the inclination slope of the cantilever at the laser spot location when the tip is displaced vertically by a unit distance [35], we normalized the resonant modes by setting the vertical displacement of the tip apex as unit one. Based on the FEM simulation, the ratio between the slope of the first-order mode and that of the second-order mode at the end of the cantilever, where the laser spot locates, is about 2.5. This suggests that the dynamic OLS of the two modes would also differ by 2.5 times, which can well account for the 2.1-fold difference in friction
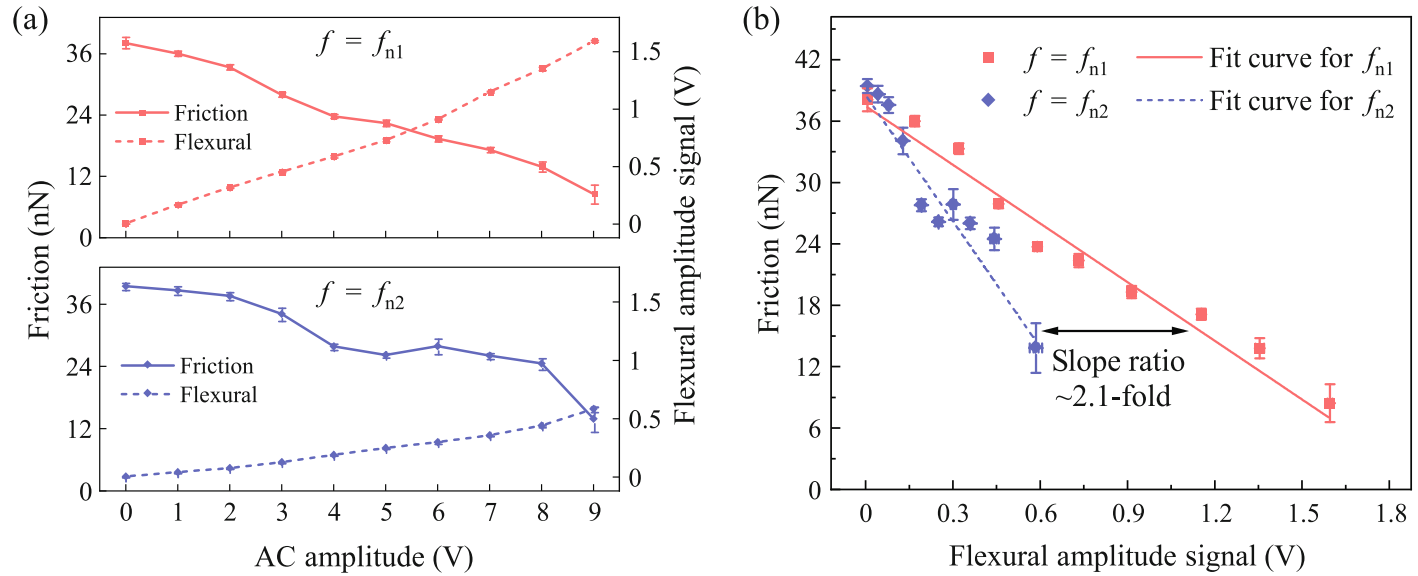

Fig. 3 (a) Variations of friction and flexural amplitude signal with actuation voltage amplitude at the first-order flexural resonance and the second-order flexural resonance, the error bar represents the standard deviation of the signal during repeated scanning under the same bias; and (b) friction versus flexural vibration amplitude signal at the first-order and the second-order flexural resonances. 
reduction rate observed in Fig. 3(b). To further validate the influence of OLS on friction reduction rate, we used a new probe and carried out two sets of friction measurements. For the first set, the laser spot was aimed at a location near the end of the cantilever as usual (see the optical image in the inset of Fig. 4(c)), and we repeated friction experiments at two resonance frequencies $f_{\mathrm{n} 1}$ and $f_{\mathrm{n} 2}$. In this case, the friction reduction rate varies 3.2-fold as frequency changed from $f_{\mathrm{nl}}$ to $f_{\mathrm{n} 2}$, as shown in Fig. 4(c). For the second set of experiment, we intentionally moved the laser spot toward the base of the cantilever (see the optical image in the inset of Fig. 4(d)) and performed similar friction experiments. Based on the simulation result in Fig. 4(b), moving the laser spot toward the base will lead to a much more significant reduction in OLS for the secondorder mode than for the first-order mode. As expected, the flexural amplitude signal at $f_{\mathrm{n} 2}$ in the second set of experiments was substantially reduced, resulting in a contrast in friction reduction rate as high as 9.4-fold. Combining the FEM simulation and the friction experiments, we conclude that vibration-induced friction reduction depends primarily on the real vertical vibration amplitude and is less sensitive to vibration frequencies or vibration modes if the OLS is carefully taken into account.

\subsection{Comparison with the $\mathrm{P}-\mathrm{T}$ model considering external excitation}

To better understand the friction reduction effect in our experiments, we performed numerical simulation using the one-dimensional $\mathrm{P}-\mathrm{T}$ model with external excitation $[12,27]$. As schematically shown in Fig. 5(a), the model describes a particle representing the AFM tip being dragged by a support via harmonic spring to slide along a periodic energy landscape. When the thermal effect is neglected, the motion of the particle can be determined by the Langevin equation as follows:

$$
m \frac{\mathrm{d}^{2} x}{\mathrm{~d} t^{2}}+m \mu \frac{\mathrm{d} x}{\mathrm{~d} t}+\frac{\partial E(x, t)}{\partial x}=0
$$

where $m$ is the mass of the particle, $x$ is the position (a)
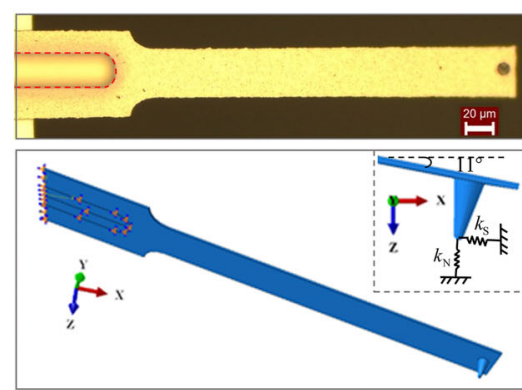

(c)

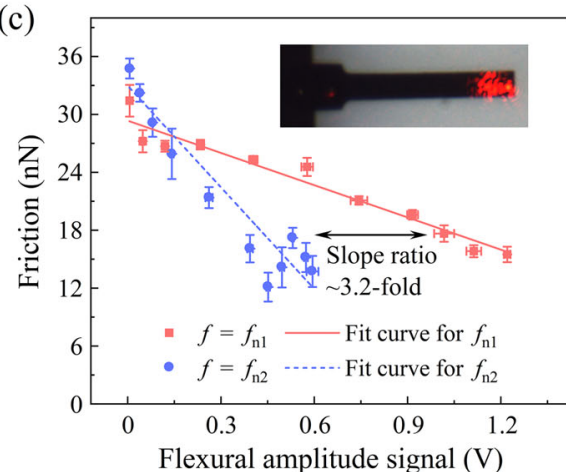

(b)

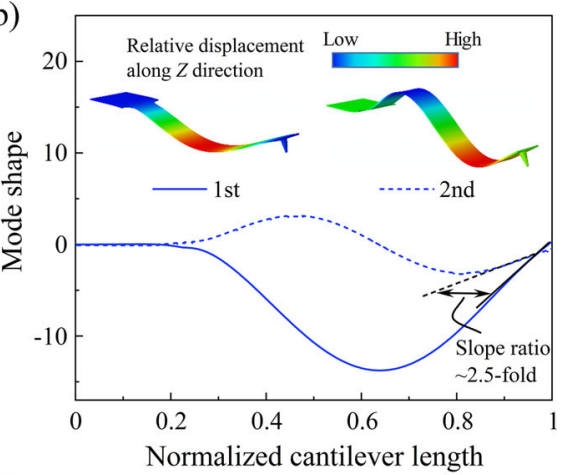

(d)

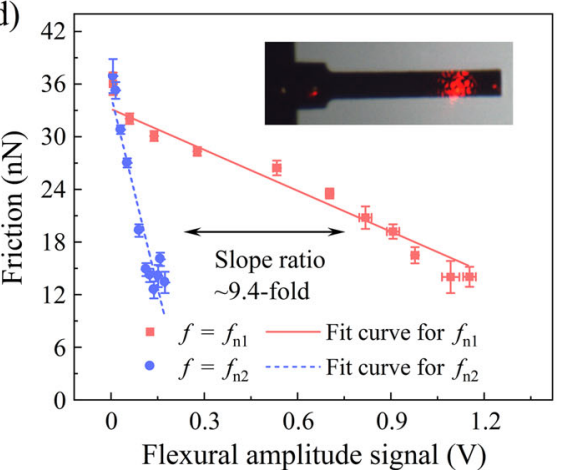

Fig. 4 FEM simulation of the micro-cantilever vibration modes and further experimental validation. (a) An optical image of the AFM probe (the upper panel) and the FEM model used in the simulation (the lower panel); (b) calculated shapes of the first-order (solid line) and the second-order (dotted line) flexural modes; and (c, d) friction versus flexural amplitude signal at the first-order and the second-order flexural resonances using a new probe when the laser spot was (c) near the edge or (d) intentionally moved toward the base, the insets are the optical images showing the position of the laser spot during different measurements, and the error bar represents the standard deviation of the data. 
of the particle, $\mu$ is the viscous damping coefficient, $t$ is the time, and $E(x, t)$ is the total potential energy. $E(x, t)$ consists of two parts, i.e., the sinusoidal interaction potential between the particle and the surface and the elastic energy of the spring:

$$
E(x, t)=-\frac{U}{2} \cos \left(\frac{2 \pi x}{a}\right)+\frac{1}{2} k(v t-x)^{2}
$$

where $U$ is the corrugation amplitude, $a$ is the lattice constant, $k$ is the effective spring constant of the system, and $v$ is the moving speed of the support. In the simulation, a fourth-order Runge-Kutta algorithm [41] was used to solve the Langevin equation numerically and the mean value $\langle F\rangle$ of the instantaneous lateral force $F=k(v t-x)$ was calculated when the particle slipped over multiple periods. Consistent with most of previous models [12, 27, 28], the effect of flexural vibration in our simulation was regarded as a perturbation to the potential energy, which oscillated with a frequency $f$ and had a relative modulation factor $\alpha$. Consequently, the perturbed potential energy $U$ becomes $U=U_{0}[1+\alpha \cos (2 \pi f t)]$, where $\alpha$ is typically assumed to depend on the flexural vibration amplitude of the probe. It should be noted that, although such approach is commonly adopted, the exact scaling relationship between $\alpha$ and the vibration amplitude has not yet been quantitatively examined.

The dynamical behavior of our system is determined by five dimensionless parameters: $\xi=\mu \sqrt{m / k}$, $\eta=2 \pi^{2} U_{0} / k a^{2}, V=v \sqrt{m / k a^{2}}, f \sqrt{m / k}$, and $\alpha$. The lattice constant $a$ of PPLN is $5.15 \AA$, and the sliding velocity $v$ was chosen as $200 \mathrm{~nm} / \mathrm{s}$ to be consistent with the experiments. The effective spring stiffness $k$ was set as $21.2 \mathrm{~N} / \mathrm{m}$ based on the calculated value from FEM simulations. As for the remaining parameters, we have chosen the following typical values as in Refs. [12, 28, 42]: $m=10^{-12} \mathrm{~kg}, U_{0}=12 \mathrm{eV}$, and $\mu=2 \sqrt{k / m}=9.2 \times 10^{6} \mathrm{~s}^{-1}$ (critical damping coefficient). The resultant dimensionless parameters are $\xi=2$, $\eta=6.7$, and $V=8.4 \times 10^{-5}$.

Figure 5(b) shows the dependence of $\langle F\rangle$ on actuation frequency for different relative amplitudes $\alpha=0.1,0.3,0.6$. Consistent with the previous findings $[12,22,27,42]$, our result indicates that friction can be suppressed only when the external excitation frequency is within an appropriate interval, i.e., $f_{\text {lower }} \leq f \leq f_{\text {upper }}$. The lower bound frequency $f_{\text {lower }}$ is the washboard frequency $v / a$ marked by the vertical dashed line in
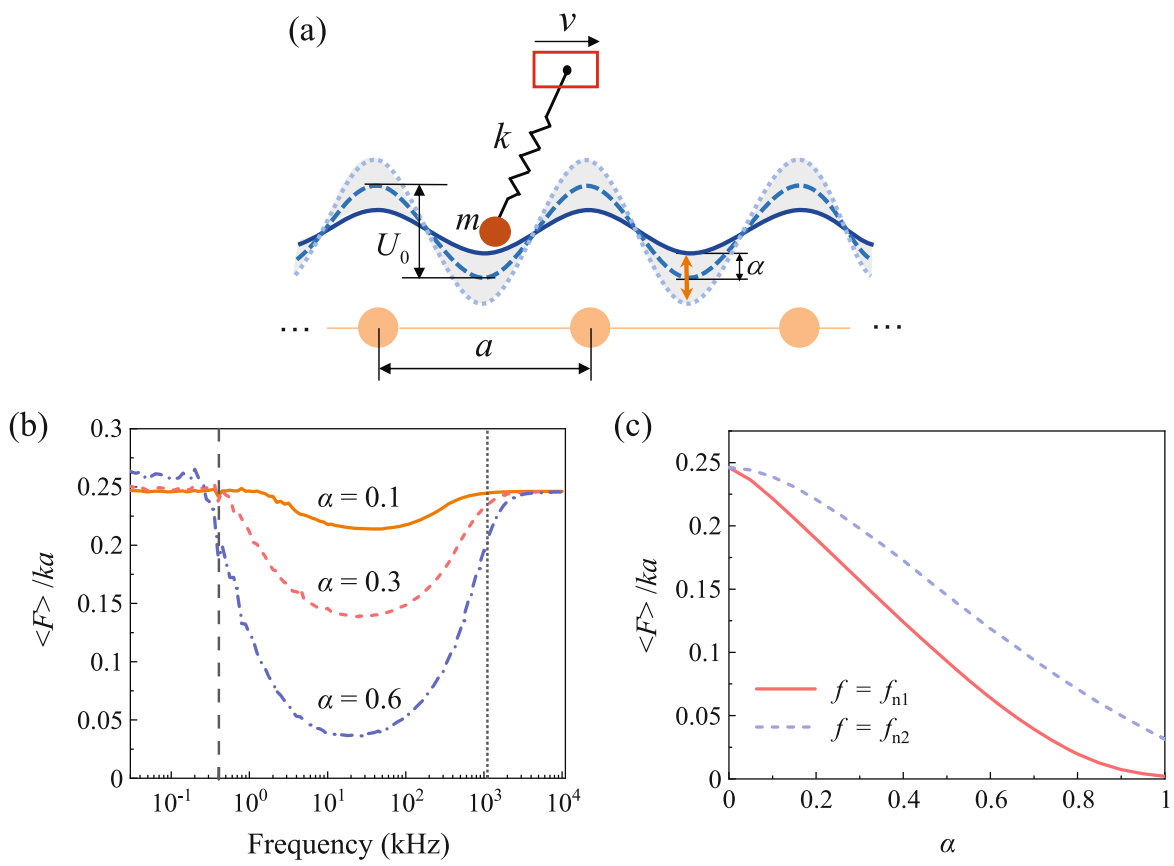

Fig. 5 Numerical simulation using the P-T friction model. (a) A schematic diagram showing the one-dimensional P-T model with a perturbed energy corrugation due to out-of-plane external actuation; (b) mean friction as a function of actuation frequency calculated for different relative perturbation amplitudes; and (c) mean friction as a function of relative perturbation amplitude calculated at the two resonant frequencies. 
Fig. 5(b), which is determined by the characteristic time scale required for the support to span one period of the interaction potential. The upper bound $f_{\text {upper }}$ is the eigen-frequency of the P-T model $f_{\mathrm{PT}}$, marked by the vertical dotted line in Fig. 5(b), which is determined by the corrugation amplitude, the mass of particle, the spring stiffness, and the slip position [42]. All actuation frequencies examined in our experiments $(1-800 \mathrm{kHz})$ are within this interval. It can be clearly seen that reduction in friction is more significant when $\alpha$ increases. For a more quantitative comparison, we calculated variation of $\langle F\rangle$ with $\alpha$ for actuation frequencies $f=f_{\mathrm{n} 1}$ and $f_{\mathrm{n} 2}$, as shown in Fig. 5(c). Under both actuation frequencies, $\langle F\rangle$ decreases approximately linearly as $\alpha$ increases with a similar slope, which is also supported by above conclusion that friction reduction rate is less dependent on vibration frequency. Combining the experimental results in Fig. 3(b), one can deduce that the relative perturbation factor $\alpha$ widely adopted in $\mathrm{P}-\mathrm{T}$ models with external excitation $[12,27,28]$ is approximately linearly proportional to the vertical vibration amplitude of the probe.

\section{Conclusions}

Based on the coupling effect between mechanical vibration and friction, we proposed a new strategy for regulating nanoscale friction by introducing a piezoelectric thin film on the contact interface. Experimentally, we have demonstrated that interfacial friction between conductive AFM probes and piezoelectric thin films could be significantly reduced (> 70\%) by imposing a low-voltage AC bias (<10 V). Such friction modulation is caused by the local out-of-plane vibration around the contact area due to the intrinsic piezoelectricity, whose amplitude is further amplified by mechanical contact resonance. Furthermore, combining experiments and numerical $\mathrm{P}-\mathrm{T}$ model simulations, we found that there existed an approximately linear correlation between the outof-plane vibration amplitude and the relative energy perturbation factor $\alpha$. As piezoelectric thin films can be easily integrated in devices, our work provides a viable approach for achieving active real-time friction modulation for small-scale interfaces without the needs of additional vibration source or global excitation that may adversely affect device functionalities.

\section{Acknowledgements}

This work was funded by the National Natural Science Foundation of China (12025203, 11772169, 11921002, and 11890671), the National Science and Technology Major Project (2017-VI-0003-0073), and the Initiative Program of State Key Laboratory of Tribology (SKLT2019B02). The authors thank Dr. Kaiyue FANG and Prof. Fei FANG both from Tsinghua University for providing the PMN-PT sample.

Electronic Supplementary Material: Supplementary material is available in the online version of this article at https://doi.org/10.1007/s40544-021-0552-y.

Open Access This article is licensed under a Creative Commons Attribution 4.0 International License, which permits use, sharing, adaptation, distribution and reproduction in any medium or format, as long as you give appropriate credit to the original author(s) and the source, provide a link to the Creative Commons licence, and indicate if changes were made.

The images or other third party material in this article are included in the article's Creative Commons licence, unless indicated otherwise in a credit line to the material. If material is not included in the article's Creative Commons licence and your intended use is not permitted by statutory regulation or exceeds the permitted use, you will need to obtain permission directly from the copyright holder.

To view a copy of this licence, visit http://creativecommons.org/licenses/by/4.0/.

\section{References}

[1] Holmberg K, Erdemir A. Influence of tribology on global energy consumption, costs and emissions. Friction 5(3): 263-284 (2017)

[2] Dowson D. History of Tribology. 2nd edn., London (UK): Professional Engineering Publishing, 1998

[3] Maboudian R, Carraro C. Surface chemistry and tribology of MEMS. Annu Rev Phys Chem 55: 35-54 (2004) 
[4] Li J J, Zhang C H, Ma L R, Liu Y H, Luo J B. Superlubricity achieved with mixtures of acids and glycerol. Langmuir 29(1): 271-275 (2013)

[5] $\mathrm{Hu}$ Y Z, Granick S. Microscopic study of thin film lubrication and its contributions to macroscopic tribology. Tribol Lett 5(1): 81-88 (1998)

[6] Hod O, Meyer E, Zheng Q S, Urbakh M. Structural superlubricity and ultralow friction across the length scales. Nature 563 (7732): 485-492 (2018)

[7] Dienwiebel M, Verhoeven G S, Pradeep N, Frenken J W M, Heimberg J A, Zandbergen H W. Superlubricity of graphite. Phys Rev Lett 92(12): 126101 (2004)

[8] Urbakh M, Meyer E. The renaissance of friction. Nat Mater 9(1): 8-10 (2010)

[9] Zhang S, Hou Y, Li S Z, Liu L Q, Zhang Z, Feng X-Q, Li Q Y. Tuning friction to a superlubric state via in-plane straining. PNAS 116(49): 24452-24456 (2019)

[10] Heuberger M, Drummond C, Israelachvili J. Coupling of normal and transverse motions during frictional sliding. $J$ Phys Chem B 102(26): 5038-5041 (1998)

[11] Kumar V C, Hutchings I M. Reduction of the sliding friction of metals by the application of longitudinal or transverse ultrasonic vibration. Tribol Int 37(10): 833-840 (2004)

[12] Socoliuc A, Gnecco E, Maier S, Pfeiffer O, Baratoff A, Bennewitz R, Meyer E. Atomic-scale control of friction by actuation of nanometer-sized contacts. Science 313(5784): 207-210 (2006)

[13] Carpick R W. Controlling friction. Science 313(5784): 184-185 (2006)

[14] Lantz M A, Wiesmann D, Gotsmann B. Dynamic superlubricity and the elimination of wear on the nanoscale. Nat Nanotechnol 4(9): 586-591 (2009)

[15] Rozman M G, Urbakh M, Klafter J. Controlling chaotic frictional forces. Phys Rev E 57(6): 7340-7343 (1998)

[16] Capozza R, Vanossi A, Vezzani A, Zapperi S. Suppression of Friction by Mechanical Vibrations. Phys Rev Lett 103(8): 085502 (2009)

[17] Guerra R, Vanossi A, Urbakh M. Controlling microscopic friction through mechanical oscillations. Phys Rev E 78(3): 036110 (2008)

[18] Gao J P, Luedtke W D, Landman U. Friction control in thin-film lubrication. $J$ Phys Chem B 102(26): 5033-5037 (1998)

[19] Cheng Y, Zhu P Z, Li R. The influence of vertical vibration on nanoscale friction: A molecular dynamics simulation study. Crystals 8(3): 129 (2018)

[20] Gnecco E, Socoliuc A, Maier S, Gessler J, Glatzel T, Baratoff A, Meyer E. Dynamic superlubricity on insulating and conductive surfaces in ultra-high vacuum and ambient environment. Nanotechnology 20(2): 025501 (2009)

[21] Gueye B, Zhang Y, Wang Y J, Chen Y F. Experimental and theoretical investigations on the nanoscale kinetic friction in ambient environmental conditions. Nano Lett 15(7): 4704-4712 (2015)

[22] Shi S, Guo D, Luo J B. Micro/atomic-scale vibration induced superlubricity. Friction 9(5): 1163-1174 (2021)

[23] Dinelli F, Biswas S K, Briggs G A D, Kolosov O V. Ultrasound induced lubricity in microscopic contact. Appl Phys Lett 71(9): 1177-1179 (1997)

[24] Jeon S, Thundat T, Braiman Y. Effect of normal vibration on friction in the atomic force microscopy experiment. Appl Phys Lett 88(21): 214102 (2006)

[25] Jiryaei Sharahi H, Egberts P, Kim S. Mechanisms of friction reduction of nanoscale sliding contacts achieved through ultrasonic excitation. Nanotechnology 30(7): 075502 (2019)

[26] Pedraz P, Wannemacher R, Gnecco E. Controlled suppression of wear on the nanoscale by ultrasonic vibrations. ACS Nano 9(9): 8859-8868 (2015)

[27] Fajardo O Y, Gnecco E, Mazo J J. Out-of-plane and in-plane actuation effects on atomic-scale friction. Phys Rev B 89(7): 075423 (2014)

[28] Iizuka H, Nakamura J, Natori A. Control mechanism of friction by dynamic actuation of nanometer-sized contacts. Phys Rev B 80(15): 155449 (2009)

[29] Steiner P, Roth R, Gnecco E, Baratoff A, Maier S, Glatzel T, Meyer E. Two-dimensional simulation of superlubricity on $\mathrm{NaCl}$ and highly oriented pyrolytic graphite. Phys Rev B 79(4): 045414 (2009)

[30] Weaver J M R. High resolution atomic force microscopy potentiometry. J Vac Sci Technol B 9(3): 1559 (1991)

[31] Damjanovic D. Ferroelectric, dielectric and piezoelectric properties of ferroelectric thin films and ceramics. Rep Prog Phys 61(9): 1267-1324 (1998)

[32] Soergel E. Piezoresponse force microscopy (PFM). J Phys D: Appl Phys 44(46): 464003 (2011)

[33] Li Q Y, Kim K S, Rydberg A. Lateral force calibration of an atomic force microscope with a diamagnetic levitation spring system. Rev Sci Instrum 77(6): 065105 (2006)

[34] Liu Y, Guo Q Q, Nie H Y, Lau W M, Yang J. Optimization and calibration of atomic force microscopy sensitivity in terms of tip-sample interactions in high-order dynamic atomic force microscopy. J Appl Phys 106(12): 124507 (2009)

[35] Liu Z, Jeong Y, Menq C H. Calibration of measurement sensitivities of multiple micro-cantilever dynamic modes in atomic force microscopy using a contact detection method. Rev Sci Instrum 84(2): 023703 (2013) 
[36] Rabe U, Janser K, Arnold W. Vibrations of free and surface-coupled atomic force microscope cantilevers: Theory and experiment. Rev Sci Instrum 67(9): 3281-3293 (1996)

[37] Tshiprut Z, Filippov A E, Urbakh M. Tuning diffusion and friction in microscopic contacts by mechanical excitations. Phys Rev Lett 95: 016101 (2005)

[38] Labuda A, Proksch R. Quantitative measurements of electromechanical response with a combined optical beam and interferometric atomic force microscope. Appl Phys Lett 106(25): 253103 (2015)

[39] Balke N, Jesse S, Yu P, Carmichael B, Kalinin S V,

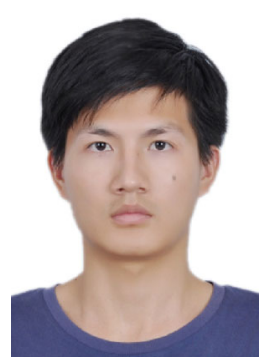

Jiawei CAO. He received his B.S. degree in 2018 from Tianjin University, China. Now he is a Ph.D. candidate in the Department

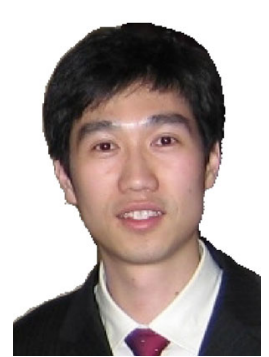

Qunyang LI. He is a professor in the Department of Engineering Mechanics at Tsinghua University. Before taking this position, he worked as a postdoctoral researcher and research scientist at the University of Pennsylvania from 2008 to 2012. He received his Ph.D. degree from the
Tselev A. Quantification of surface displacements and electromechanical phenomena via dynamic atomic force microscopy. Nanotechnology 27(42): 425707 (2016)

[40] Gannepalli A, Yablon D G, Tsou A H, Proksch R. Mapping nanoscale elasticity and dissipation using dual frequency contact resonance AFM. Nanotechnology 22(35): 355705 (2011)

[41] Kasdin N J. Runge-kutta algorithm for the numerical integration of stochastic differential equations. J Guid Control Dyn 18(1): 114-120 (1995)

[42] Dong Y L, Vadakkepatt A, Martini A. Analytical models for atomic friction. Tribol Lett 44(3): 367-386 (2011)

of Engineering Mechanics at Tsinghua University. His current research interest is nano-tribology of smart materials.

Brown University in 2008 and B.S. and M.S. degrees in engineering mechanics from Tsinghua University in 2001 and 2003, respectively. His current research aims at understanding how surfaces interact at small scales and bridging nanoscale intrinsic properties to macroscale behaviors through experiments and mechanics modeling. 